\title{
Charge pumping driven by the laser-induced dynamics of the exchange splitting
}

\author{
Frank Freimuth, ${ }^{*}$ Stefan Blügel, and Yuriy Mokrousov \\ Peter Grünberg Institut and Institute for Advanced Simulation, Forschungszentrum Jülich and JARA, 52425 Jülich, Germany
}

(Received 8 December 2016; revised manuscript received 5 March 2017; published 31 March 2017)

\begin{abstract}
We show that electric currents are induced in inversion asymmetric ferromagnets if the exchange splitting varies in time after excitation by laser pulses. We interpret this phenomenon as the magnetic variant of the inverse Edelstein effect. Based on ab initio calculations we determine the size of this effect in $\mathrm{Co} / \mathrm{Pt}(111)$ and $\mathrm{Mn} / \mathrm{W}(001)$ magnetic bilayer systems and investigate its dependence on the magnetization direction. The comparison of our theoretical results to experiments measuring the $\mathrm{THz}$ signal emitted after laser excitation suggests that ultrafast demagnetization in $3 d$ transition metal ferromagnets is dominated by transverse spin fluctuations rather than by a reduction of the exchange splitting.
\end{abstract}

DOI: 10.1103/PhysRevB.95.094434

\section{INTRODUCTION}

Optical excitation of magnetic heterostructures such as $\mathrm{Fe} / \mathrm{Au}$ or $\mathrm{Co} / \mathrm{Pt}$ by femtosecond (fs) laser pulses triggers in-plane subpicosecond electric currents [1-3]. Two distinct mechanisms of this effect have been identified already: First, a superdiffusive spin current is launched, which propagates from the magnetic layer into the nonmagnetic metal [4-6]. The generation of this superdiffusive spin current can be interpreted as an ultrafast nonequilibrium variant of the spin-dependent Seebeck effect triggered by the laser-induced heating [7]. The inverse spin Hall effect (ISHE) converts this subpicosecond spin current into a charge current pulse flowing parallel to the bilayer interface $[1,3]$. Second, when a circularly polarized laser beam is used, an additional effect can be observed: The magnetization of the magnetic layer tilts because of the effective magnetic field generated by the inverse Faraday effect or by the optical spin-transfer torque [8-10]. Via charge pumping due to the inverse spin-orbit torque (ISOT) [11-13] the tilting magnetization induces an interfacial electric current pulse [2].

These in-plane subpicosecond photocurrents can be quantified contactlessly by measuring the resulting emission of terahertz (THz) electromagnetic radiation $[1,2]$. By optimizing the composition and layer thicknesses of the magnetic heterostructures one can even achieve $\mathrm{THz}$ radiation that is sufficiently strong to make photocurrents in magnetic heterostructures attractive for application in table-top ultrabroadband $\mathrm{THz}$ emitters [3]. Additionally, the photocurrents might provide a new and complementary tool to investigate magnetization dynamics at subpicosecond time scales, where other magnetooptical probing techniques face difficulties [14-16].

Another effect triggered by fs laser pulses is ultrafast demagnetization [17]. Several experiments and theories suggest that ultrafast demagnetization is accompanied by a breakdown of the local magnetic moments and by a collapse of the exchange splitting [18-21]. Since the electric conductivity tensor is affected by such electronic structure changes one can use measurements of the electric conductivity tensor in order to assess to which extent ultrafast demagnetization is accompanied by a reduction of the exchange splitting [22].

\footnotetext{
*Corresponding author: f.freimuth@fz-juelich.de
}

In such experiments only small changes of the conductivity have been found in 50-nm-thick $\mathrm{Fe}(001) / \mathrm{MgO}(001)$ films. Furthermore, for unsupported Ni monolayers, it is found from exact time propagation of a many-electron small-cluster model that laser-induced demagnetization arises dominantly from fluctuations of the orientations of the local magnetic moments, while the local spin polarization is not reduced significantly [23]. In contrast, in the strong-field limit, which can be realized by reducing the laser-pulse duration while keeping the fluence constant, it has been predicted that the local magnetic moments are reduced and that no fluctuations of the orientations of the local magnetic moments are excited [21]. Additionally, it is expected that the extent to which a reduction of the exchange splitting contributes to ultrafast demagnetization is material dependent: When the local magnetic moments are particularly stable, a collapse of the exchange splitting is less likely than fluctuations of the orientations of the local magnetic moments [23].

Moreover, whether ultrafast demagnetization is dominated by transverse fluctuations or by a reduction of the local magnetic moments might also depend on the geometry. Superdiffusive spin currents, which carry magnetization away from the magnetic layers, provide a nonlocal transport contribution to ultrafast demagnetization in metallic heterostructures, which adds to the demagnetization due to the local spin-flip processes mediated by the spin-orbit interaction (SOI) $[4,5,7,21,24]$. In heterostructures composed of two $\mathrm{Co} / \mathrm{Pt}$ multilayers separated by a $\mathrm{Ru}$ spacer the contribution of the superdiffusive spin currents to the total amount of demagnetization has been estimated at $25 \%$ [4]. In $\mathrm{Ni} / \mathrm{Ru} / \mathrm{Fe}$ heterostructures $30 \%$ of demagnetization of $\mathrm{Ni}$ is attributed to superdiffusive spin currents flowing from $\mathrm{Fe}$ to $\mathrm{Ni}$ [25]. The semiclassical theory seems to suggest that demagnetization by superdiffusive spin currents goes hand in hand with a reduction of local spin polarization, at least initially. However, it has been pointed out that even in this case the system will quickly reach a state where the local magnetic moments are tilted against each other while they have the same modulus as in equilibrium [26].

Since small-angle precession of the magnetization at $\mathrm{GHz}$ frequencies, i.e., the coherent dynamics of the transverse degree of freedom, generates measurable electrical currents [13] as well as heat currents [27,28] in metallic ferromagnets with inversion asymmetry, the question arises whether also the subpicosecond reduction of the local spin polarization, i.e., the 
dynamics of the longitudinal degree of freedom, induces such currents. The idea that the dynamics of the exchange splitting can induce measurable charge or heat currents seems realistic in view of the strong formal similarities with the inverse Edelstein effect, which consists in the conversion of spin currents flowing towards interfaces or surfaces into transverse charge currents $[29,30]$. In order to calculate the inverse Edelstein effect, which results from inversion asymmetry and SOI at interfaces or surfaces, one can describe the spin injection into the interface or surface by a magnetic field that varies linearly in time [31]. Experiments on the inverse Edelstein effect are usually performed on nonmagnetic interfaces such as $\mathrm{Bi} / \mathrm{Ag}$ [29] and $\mathrm{Cu} / \mathrm{Bi}$ [30]. Here we are interested in magnetic bilayer systems such as $\mathrm{Co} / \mathrm{Pt}$ or $\mathrm{Mn} / \mathrm{W}$, where the exchange splitting is time dependent after excitation by a fs laser pulse. Since a time-dependent magnetic field induces charge currents in $\mathrm{Bi} / \mathrm{Ag}$ and $\mathrm{Cu} / \mathrm{Bi}$, it is natural to expect that the dynamics of the exchange splitting induces charge currents in $\mathrm{Co} / \mathrm{Pt}$ and $\mathrm{Mn} / \mathrm{W}$ magnetic bilayers as well. The generation of electric currents by time-dependent magnetic fields in nonmagnetic materials is also known as gyrotropic magnetic effect [32].

If a dynamical exchange splitting drives charge currents, this effect can be employed in experiments in order to investigate the question whether ultrafast demagnetization is dominated by transverse spin fluctuations or a reduction of the local magnetic moments depending on the magnetic material, the laser pulse characteristics, and the geometry. In this paper we determine the charge current driven by a dynamical exchange splitting in $\mathrm{Co} / \mathrm{Pt}(111)$ and $\mathrm{Mn} / \mathrm{W}(001)$ magnetic bilayers based on ab initio density functional theory calculations. This paper is structured as follows: In Sec. II we introduce our computational approach, which uses the Kubo linear-response formalism in order to calculate the charge pumping by a dynamical exchange splitting. In Sec. III we present the results for $\mathrm{Co} / \mathrm{Pt}(111)$ and $\mathrm{Mn} / \mathrm{W}(001)$ magnetic bilayers, we compare to recent experiments measuring the $\mathrm{THz}$ emission from similar bilayer systems under laser irradiation, and we discuss implications of this theory-experiment comparison for the ultrafast demagnetization process in these bilayer systems. We conclude with a summary in Sec. IV.

\section{FORMALISM}

Within the local spin density approximation (LSDA) interacting many-electron systems in equilibrium are described by the effective single-particle Hamiltonian

$$
H(\boldsymbol{r})=H_{0}(\boldsymbol{r})-\boldsymbol{m} \cdot \hat{\boldsymbol{M}} \Omega^{\mathrm{xc}}(\boldsymbol{r}) .
$$

Here $H_{0}$ contains kinetic energy, scalar potential, and SOI. The second term on the right-hand side describes the exchange interaction in collinear magnets. $\hat{M}$ is a normalized vector that points into the magnetization direction. $\boldsymbol{m}=-\mu_{\mathrm{B}} \sigma$ is the spin magnetic moment operator, where $\mu_{\mathrm{B}}$ is the Bohr magneton and $\sigma=\left(\sigma_{x}, \sigma_{y}, \sigma_{z}\right)^{\mathrm{T}}$ is the vector of Pauli matrices. The exchange field $\Omega^{\mathrm{xc}}(\boldsymbol{r})=\frac{1}{2 \mu_{\mathrm{B}}}\left[V_{\text {minority }}^{\text {eff }}(\boldsymbol{r})-V_{\text {majority }}^{\text {eff }}(\boldsymbol{r})\right]$ is given by the difference between the effective potentials of minority and majority electrons.

When the local spin polarization goes down during ultrafast demagnetization also the exchange splitting is reduced. We model this by the time-dependent Hamiltonian

$$
\begin{aligned}
H(\boldsymbol{r}, t) & =H_{0}(\boldsymbol{r})-\boldsymbol{m} \cdot \hat{\boldsymbol{M}} \Omega^{\mathrm{xc}}(\boldsymbol{r}) \eta(t) \\
& =H_{0}(\boldsymbol{r})+\mu_{\mathrm{B}} \Omega_{\|}^{\mathrm{xc}}(\boldsymbol{r}) \eta(t),
\end{aligned}
$$

where $\eta(t)$ describes the reduction of the exchange field and we defined the operator $\Omega_{\|}^{\mathrm{xc}}(\boldsymbol{r})=\boldsymbol{\sigma} \cdot \hat{\boldsymbol{M}} \Omega^{\mathrm{xc}}(\boldsymbol{r})$. We assume that the exchange field is reduced linearly in time at a rate of $\gamma$, i.e., $d \eta / d t=-\gamma<0$. This induces an electrical current per unit length of

$$
J_{\alpha}=-L_{\alpha} \frac{\gamma}{A}
$$

where $A$ is the area of the unit cell and we defined the response coefficients

$$
L_{\alpha}=e \mu_{\mathrm{B}} \lim _{\omega \rightarrow 0} \frac{\operatorname{Im} G_{v_{\alpha}, \Omega_{\|}^{\mathrm{xc}}}^{\mathrm{R}}(\hbar \omega)}{\hbar \omega} .
$$

Here $\alpha=x, y, z$ denotes the Cartesian component, $e>0$ is the elementary positive charge, and

$$
G_{v_{\alpha}, \Omega_{\|}^{\mathrm{xc}}}^{\mathrm{R}}(\hbar \omega)=-i \int_{0}^{\infty} d t e^{i \omega t}\left\langle\left[v_{\alpha}(t), \Omega_{\|}^{\mathrm{xc}}(0)\right]_{-}\right\rangle
$$

is the retarded correlation function describing the response of the velocity operator $\boldsymbol{v}$ ( $v_{\alpha}$ is the $\alpha$ th Cartesian component) to perturbations by the operator $\Omega_{\|}^{\mathrm{xc}}$.

Our choice to define the current density as a current per length in Eq. (3) is convenient for bilayer systems, where the current distribution is inhomogeneous along the stacking direction. The current density $j_{\alpha}$ (current per area) used to describe periodic bulk systems in three dimensions is related to $J_{\alpha}$ (current per length) by

$$
J_{\alpha}=\int d z j_{\alpha}(z),
$$

where $z$ is the coordinate along the stacking direction, which is perpendicular to the bilayer interface.

The coefficient $L_{\alpha}$ defined in Eq. (4) describes only the electric current induced by the dynamical exchange splitting and consequently $J_{\alpha}$ given by Eq. (3) contains only this current and misses several contributions that we discussed in the Introduction, namely the current from the conversion of the superdiffusive spin current into an interfacial charge current by the inverse spin Hall effect and the current from the inverse spin-orbit torque and the tilting magnetization due to the inverse Faraday effect. The theoretical modeling of these two currents has been described already elsewhere $[3,16]$.

The details of the laser pulse that triggers ultrafast demagnetization do not enter the expression for the current $J_{\alpha}$ given by Eq. (3), only the rate of change of the exchange field matters. This is a consequence of our model, Eq. (2), which only phenomenologically describes the reduction of the exchange splitting during ultrafast demagnetization but does not model the mechanism of ultrafast demagnetization itself. In order to apply our theory to a given experimental setup it is therefore necessary to determine the parameter $\gamma$ from measurements of ultrafast demagnetization. In Sec. III we will provide an example of this procedure. 
For the magnetic bilayer systems considered in this work, Eq. (4) can be rewritten as

$$
L_{\alpha}=-\frac{e \hbar}{\pi \mathcal{N}} \sum_{k n m} \frac{\mu_{\mathrm{B}} \Gamma^{2} \operatorname{Re}\left[\left\langle\psi_{\boldsymbol{k} n}\left|v_{\alpha}\right| \psi_{\boldsymbol{k} m}\right\rangle\left\langle\psi_{\boldsymbol{k} m}\left|\Omega_{\|}^{\mathrm{xc}}\right| \psi_{\boldsymbol{k} n}\right\rangle\right]}{\left[\left(\mathcal{E}_{\mathrm{F}}-\mathcal{E}_{\boldsymbol{k} n}\right)^{2}+\Gamma^{2}\right]\left[\left(\mathcal{E}_{\mathrm{F}}-\mathcal{E}_{\boldsymbol{k} m}\right)^{2}+\Gamma^{2}\right]},
$$

where the Bloch state $\left|\psi_{k n}\right\rangle$ and the band energy $\mathcal{E}_{\boldsymbol{k} n}$ satisfy $H\left|\psi_{k n}\right\rangle=\mathcal{E}_{k n}\left|\psi_{k n}\right\rangle$ with the Hamiltonian $H$ defined in Eq. (1) and we use the constant broadening $\Gamma$ of the electronic bands in order to describe disorder. $\mathcal{E}_{\mathrm{F}}$ is the Fermi energy and $\mathcal{N}$ is the number of $\boldsymbol{k}$ points used to sample the Brillouin zone. $L_{\alpha}$ in Eq. (7) depends on the magnetization direction $\hat{\boldsymbol{M}}$ and is odd in $\hat{\boldsymbol{M}}$, i.e., $L_{\alpha}(\hat{\boldsymbol{M}})=-L_{\alpha}(-\hat{\boldsymbol{M}})$. Generally, Eq. (4) can also contain an even component, which we do not include in Eq. (7). However, due to mirror and rotational symmetries this even component vanishes in the bilayer systems considered in this work.

$L_{\alpha}$ describes the coupling between a polar vector and a scalar and therefore $L_{\alpha}$ is nonzero only in systems with inversion asymmetry. Furthermore, $L_{\alpha}$ is zero when SOI is not included in the Hamiltonian.

The broadening $\Gamma$ is related to the lifetime $\tau$ of the electronic states by the expression $\tau=\hbar /(2 \Gamma)$. The lifetime can be controlled in experiments by modifying the amount of disorder or the temperature. The electrical conductivity is also sensitive to the lifetime. Measurements of the electrical conductivity can be used to extract the lifetime and from this the broadening $\Gamma$ needed in our theoretical description. Equation (7) is valid in the static limit. It describes the electric current induced by a dynamical exchange splitting correctly when the condition $\gamma \ll \tau^{-1}$ is satisfied.

\section{AB INITIO RESULTS AND DISCUSSION}

In the following we use $a b$ initio density functional theory calculations in order to investigate the electric current driven by the dynamical exchange splitting in $\mathrm{Mn} / \mathrm{W}(001)$ and $\mathrm{Co} / \mathrm{Pt}(111)$ magnetic bilayers. Both $\mathrm{Pt}$ and $\mathrm{W}$ provide large SOI at the interfaces, which is ideal in order to maximize the effect. In a previous work we studied in these two systems already the spin-orbit torques (SOTs), which also require SOI and inversion asymmetry and are therefore loosely related to the effect of interest here. Laser-induced electric currents have been studied experimentally in $\mathrm{Co} / \mathrm{Pt}$ already [16], while such experiments have not yet been performed in $\mathrm{Mn} / \mathrm{W}(001)$. However, monolayers of $\mathrm{Mn}$ on W(001) have already been prepared experimentally and their magnetic structure has been determined from spin-polarized scanning tunneling microscopy [33].

In our calculations, the $\mathrm{Mn} / \mathrm{W}(001)$ system is composed of one monolayer of $\mathrm{Mn}$ deposited on nine atomic layers of $\mathrm{W}(001)$ and the $\mathrm{Co} / \mathrm{Pt}(111)$ bilayer consists of three atomic layers of Co deposited on ten atomic layers of $\mathrm{Pt}(111)$. Details of the electronic structure calculations are given in [34]. The ground-state magnetic structure of $\mathrm{Mn} / \mathrm{W}(001)$ is a spin spiral [33]. However, in the present work we treat $\mathrm{Mn} / \mathrm{W}(001)$ as a ferromagnet. We use Eq. (7) in order to calculate the response coefficient $L_{\alpha}$, where we employ Wannier interpolation for
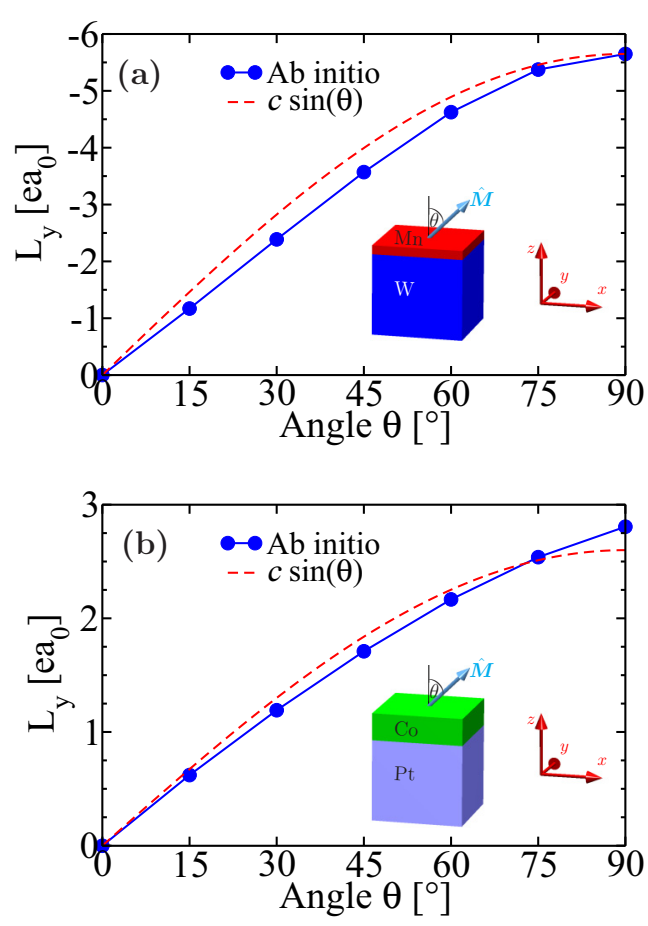

FIG. 1. Response coefficient $L_{y}$ vs polar angle $\theta$ of the magnetization direction in (a) the $\mathrm{Mn} / \mathrm{W}(001)$ magnetic bilayer and (b) the $\mathrm{Co} / \mathrm{Pt}(111)$ magnetic bilayer. Circles: $A b$ initio results. Dashed line: Fit with $c \sin (\theta)$, where $c$ is the fitting parameter. $L_{y}$ is plotted in units of $e a_{0}=8.478 \times 10^{-30} \mathrm{C} \mathrm{m}$, where $e$ is the elementary positive charge and $a_{0}$ is Bohr's radius. The quasiparticle broadening is set to $\Gamma=25 \mathrm{meV}$. The insets illustrate the geometry.

computational efficiency [35,36]. Technical details on the Wannier interpolation are given in [34].

In Fig. 1 we show the coefficient $L_{y}$ as a function of the polar angle $\theta$ of the magnetization direction $\hat{\boldsymbol{M}}=(\sin \theta, 0, \cos \theta)^{\mathrm{T}}$. The angular dependence of $L_{y}$ is approximately given by $\sin \theta$, i.e., $L_{y}$ is roughly proportional to the $x$ component of $\hat{\boldsymbol{M}}$. The component $L_{x}$ (not shown in the figure) is zero in this case, because it is proportional to the $y$ component of $\hat{\boldsymbol{M}}=(\sin \theta, 0, \cos \theta)^{\mathrm{T}}$, which is zero. This angular dependence agrees to the one of the inverse Edelstein effect [29-31], which is given by $J^{\mathrm{IE}} \propto \hat{\boldsymbol{z}} \times \dot{\boldsymbol{B}}$ : In the case of the inverse Edelstein effect the in-plane electric current flowing into $y$ direction is proportional to the rate of change of the $x$ component of an applied time-dependent magnetic field, or, equivalently, it is proportional to the $x$ component of the spin injected into the interface due to a spin current flowing towards the bilayer interface. The inverse Edelstein effect is a consequence of Rashba-type SOI, which orients the spin $S$ of the electrons perpendicularly to the $\boldsymbol{k}$ vector: $\boldsymbol{S} \propto \hat{z} \times \boldsymbol{k}$. In the bilayer systems considered in this work the $\boldsymbol{k}$-linear term of the spin-orbit field is given by Rashba SOI. Since the charge pumping driven by a dynamical exchange splitting exhibits the same angular dependence as the inverse Edelstein effect, it can indeed be interpreted as a ferromagnetic variant of the inverse Edelstein effect, as anticipated in the Introduction.

In Fig. 2 we show $L_{y}$ as a function of the quasiparticle broadening $\Gamma$, which we use to model the disorder. $L_{y}$ depends 

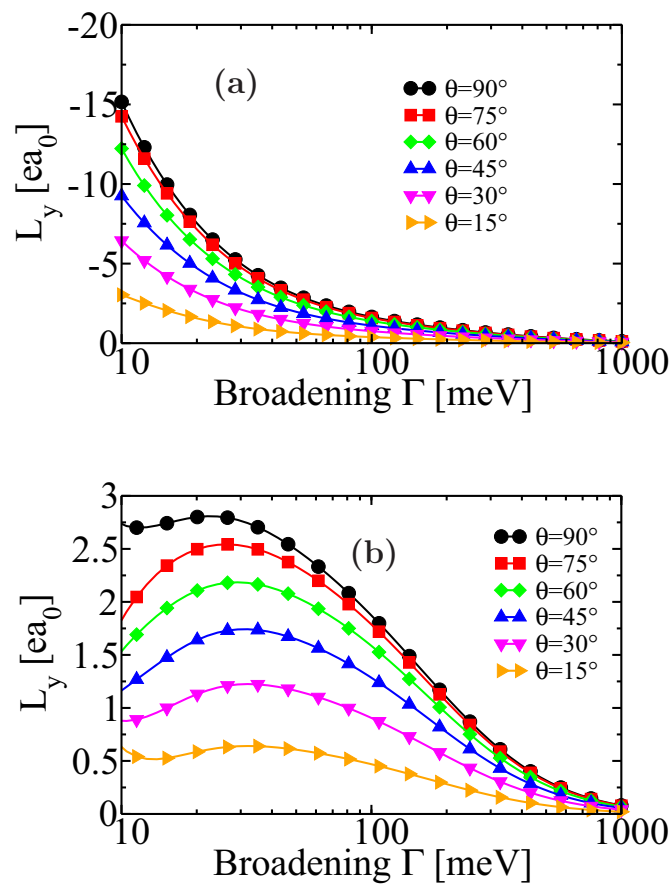

FIG. 2. Response coefficient $L_{y}$ vs quasiparticle broadening $\Gamma$ in (a) the $\mathrm{Mn} / \mathrm{W}(001)$ magnetic bilayer and (b) the $\mathrm{Co} / \mathrm{Pt}(111)$ magnetic bilayer. Results are shown for various polar angles $\theta$ of the magnetization direction.

strongly on $\Gamma$ and vanishes for very large disorder. Previously we reported a similarly strong disorder dependence of the spin-orbit torques (SOTs) in these bilayer systems [34]. In the considered $\Gamma$ range from 10 to $1000 \mathrm{meV}$ the coefficient $L_{y}$ decreases monotonically in $\mathrm{Mn} / \mathrm{W}(001)$, while it exhibits a maximum in $\mathrm{Co} / \mathrm{Pt}(111)$. A similar qualitative difference in the $\Gamma$ dependence between $\mathrm{Mn} / \mathrm{W}(001)$ and $\mathrm{Co} / \mathrm{Pt}(111)$ is found in the odd component of the SOT [34]. In order to explain these similarities we assume that the inverse Edelstein effect arises from the interfacial spin-orbit coupling in these bilayer systems, like the odd component of the SOT. Additionally, similarly to the odd component of the SOT, the inverse Edelstein effect is dominated by intraband terms for small values of $\Gamma$, while interband terms are activated when $\Gamma$ is increased.

When the magnetization direction lies in the $x z$ plane, i.e., when $\hat{\boldsymbol{M}}=(\sin \theta, 0, \cos \theta)^{\mathrm{T}}$, the $y$ component of the interfacial electric current generated when a laser-pulse is applied to the system is expected to consist of two contributions. One contribution arises from the dynamical exchange splitting and is given by $J_{y}=-L_{y} \gamma / A \propto \sin (\theta) \gamma$ as discussed above. The second contribution arises from the conversion of superdiffusive spin currents into interfacial charge currents by the ISHE [1]. The spin-polarization vector of the superdiffusive spin current is coupled to the magnetization direction $\hat{\boldsymbol{M}}$. Thus, the amount of spin current converted into charge current $J_{y}$ by ISHE is proportional to $\sin (\theta)$ like $L_{y}$. Since the two contributions to $J_{y}$ have therefore the same $\theta$ dependence they are difficult to separate experimentally. In order to reveal a relation between dynamical exchange splitting and charge currents in experiments unambiguously one possibility is to design the experiment such that the contribution from the superdiffusive spin current is suppressed. It seems likely that this can be achieved in thin magnetic layers grown on insulators. Alternatively, instead of suppressing the superdiffusive spin current one can also use magnets with bulk inversion asymmetry and identify the bulk contribution to the induced electric current. When the half-Heusler NiMnSb is tetragonally strained the $\boldsymbol{k}$-linear term in the spin-orbit field is of Dresselhaus symmetry [37]. Thereby, one can achieve that the electric current driven by the dynamical exchange splitting has a different angular dependence than the electric current from the conversion of the superdiffusive spin current. For example, one can achieve that the current driven by the dynamical exchange splitting flows in $x$ direction, i.e., $J_{x}=-L_{x} \gamma / A \propto \sin (\theta) \gamma$, when $\hat{\boldsymbol{M}}=(\sin \theta, 0, \cos \theta)^{\mathrm{T}}$.

In a recent experiment the $\mathrm{THz}$ emission from $\mathrm{CoFeB} / \mathrm{HM}$ magnetic bilayers under laser illumination was measured for several different choices of the heavy metal (HM) layer, namely $\mathrm{Cr}, \mathrm{Pd}, \mathrm{Ta}, \mathrm{W}, \mathrm{Ir}$, and Pt [3]. Thereby, it was found that when $\theta=90^{\circ}$ the interfacial electric current in $y$ direction, $J_{y}$, is proportional to the spin Hall conductivity and results from the excitation of superdiffusive spin currents that are converted into in-plane charge currents by the ISHE. This interpretation is also consistent with the Pt-thickness dependence of $J_{y}$, which suggests that $J_{y}$ is not a pure interface effect but that the Pt-thickness dependence is determined by the spin diffusion length, which governs the decay of the superdiffusive spin current in the HM layer [2,3]. Since the electric current driven by the dynamical exchange splitting is not related to the ISHE, these experimental observations imply that the contribution from the dynamical exchange splitting to $J_{y}$ must be at least one order of magnitude smaller than the contribution from the superdiffusive spin current and the ISHE, because otherwise it would have been observed in these experiments.

Based on our $a b$ initio results for the coefficient $L_{y}$ we can assess the expected order of magnitude of the electric current induced by a dynamical exchange splitting for various scenarios of demagnetization. For $50 \mathrm{fs}$ laser pulses with central wavelength $800 \mathrm{~nm}$ and fluence $1 \mathrm{~mJ} / \mathrm{cm}^{2}$ we estimate $\gamma \approx 2 \times 10^{11} \mathrm{~s}^{-1}$ in Co from the magnetization dynamics extracted from MOKE measurements [38] when we assume the scenario that no transverse fluctuations are excited and that the demagnetization corresponds to a reduction of the local magnetic moments. Previously we found that a broadening of $\Gamma=25 \mathrm{meV}$ can be used to simulate room-temperature SOT experiments on $\mathrm{Co} / \mathrm{Pt}$ bilayer systems [34]. At $\Gamma=25 \mathrm{meV}$ and $\theta=90^{\circ}$ we obtain $L_{y}=2.8 e a_{0}$ in $\mathrm{Co} / \mathrm{Pt}(111)$ in our calculations. In this scenario of demagnetization the resulting current per length driven by the dynamical exchange splitting would be $J_{y}=-71.35 \mathrm{~A} / \mathrm{m}$, which is even larger than the current $J_{y}=32 \mathrm{~A} / \mathrm{m}$ measured experimentally in $\operatorname{Co}(10 \mathrm{~nm}) / \operatorname{Pt}(2 \mathrm{~nm})$ for the laser pulse parameters given above [2]. Since the experimentally observed current $J_{y}$ has been demonstrated to arise dominantly from superdiffusive spin currents combined with ISHE [1,3], we conclude that the assumption that ultrafast demagnetization in Co corresponds to a reduction of the local magnetic moments without excitation of transverse fluctuations has to be incorrect. This finding is consistent with the experimental observation that electric conductivity is not strongly modified during ultrafast 
demagnetization in $\mathrm{Fe}(001) / \mathrm{MgO}(001)$, while any strong reduction of the exchange splitting is expected to result in a significant variation of electric conductivity [22]. Our finding is also consistent with theoretical work on ultrafast demagnetization, which reports that it is dominated by transverse spin fluctuations [23].

The electric current driven by transverse spin fluctuations has been estimated to be much smaller than the electric current from the conversion of the superdiffusive spin current [1]. If we assume the scenario that ultrafast demagnetization is dominated by spin fluctuations and that the reduction of the local magnetic moments contributes at most $5 \%$ to the total demagnetization the estimated reduction rate of the exchange splitting is at most $5 \%$ of $2 \times 10^{11} \mathrm{~s}^{-1}$, i.e., $\gamma<10^{10} \mathrm{~s}^{-1}$ in Co for the laser parameters given above. In this scenario of demagnetization the electric current $J_{y}$ driven by the dynamical exchange splitting is smaller than $3.6 \mathrm{~A} / \mathrm{m}$ in $\mathrm{Co} / \mathrm{Pt}(111)$ according to our calculations, which is one order of magnitude smaller than the experimentally measured $J_{y}$. Such a small contribution to $J_{y}$ from the dynamical exchange splitting is consistent with the experimental observation that $J_{y}$ is clearly dominated by the contribution from the superdiffusive spin current converted by the ISHE.

For the laser-pulse parameters used in the experiment, i.e., duration $50 \mathrm{fs}$ and fluence $1 \mathrm{~mJ} / \mathrm{cm}^{2}$, we can thus rule out by comparing our theoretical calculations to experiment that ultrafast demagnetization in Co dominantly arises from a reduction of the modulus of the local magnetic moments without excitation of transverse fluctuations. For shorter laser pulse duration and higher intensities a reduction of the modulus of the local magnetic moments without excitation of transverse fluctuations has been predicted from time-dependent density functional theory studies [21]. By performing measurements of the THz radiation under laser excitation with shorter laser pulses and higher intensities one should therefore be able to observe a contribution to the $\mathrm{THz}$ signal from a dynamical exchange splitting.

\section{SUMMARY}

We demonstrate that a dynamical exchange splitting induces measurable electric currents in magnetic bilayer systems with inversion asymmetry and spin-orbit interaction. Using an $a b$ initio approach, we study this effect in $\mathrm{Mn} / \mathrm{W}(001)$ and $\mathrm{Co} / \mathrm{Pt}(111)$ magnetic bilayers. The strong disorder dependence is reminiscent of the odd component of the spin-orbit torque in these magnetic bilayers pointing at the interfacial spinorbit interaction as common mechanism. The dependence on magnetization direction suggests we view electric currents driven by a dynamical exchange splitting as a ferromagnetic variant of the inverse Edelstein effect. We compare our theoretical results to experiments measuring the $\mathrm{THz}$ emission from magnetic bilayer systems under laser illumination. This leads us to the conclusion that when ultrafast demagnetization in Co is triggered by $800 \mathrm{~nm} 50$ fs laser pulses this ultrafast demagnetization is not dominated by a reduction of the local magnetic moments, suggesting an important role of transverse spin fluctuations.

\section{ACKNOWLEDGMENTS}

We gratefully acknowledge computing time on the supercomputers of Jülich Supercomputing Center and RWTH Aachen University as well as financial support from the programme SPP 1538 Spin Caloric Transport of the Deutsche Forschungsgemeinschaft.
[1] T. Kampfrath, M. Battiato, P. Maldonado, G. Eilers, J. Nötzold, S. Mährlein, V. Zbarsky, F. Freimuth, Y. Mokrousov, S. Blügel et al., Nat. Nanotechnol. 8, 256 (2013).

[2] T. J. Huisman, R. V. Mikhaylovskiy, J. D. Costa, F. Freimuth, E. Paz, J. Ventura, P. P. Freitas, S. Blügel, Y. Mokrousov, T. Rasing et al., Nat. Nanotechnol. 11, 455 (2016).

[3] T. Seifert, S. Jaiswal, U. Martens, J. Hannegan, L. Braun, P. Maldonado, F. Freimuth, A. Kronenberg, J. Henrizi, I. Radu et al., Nat. Photon. 10, 483 (2016).

[4] G. Malinowski, F. Dalla Longa, J. H. H. Rietjens, P. V. Paluskar, R. Huijink, H. J. M. Swagten, and B. Koopmans, Nat. Phys. 4, 855 (2008).

[5] M. Battiato, K. Carva, and P. M. Oppeneer, Phys. Rev. Lett. 105, 027203 (2010).

[6] A. Melnikov, I. Razdolski, T. O. Wehling, E. T. Papaioannou, V. Roddatis, P. Fumagalli, O. Aktsipetrov, A. I. Lichtenstein, and U. Bovensiepen, Phys. Rev. Lett. 107, 076601 (2011).

[7] M. Battiato, K. Carva, and P. M. Oppeneer, Phys. Rev. B 86, 024404 (2012).

[8] A. V. Kimel, A. Kirilyuk, P. A. Usachev, R. V. Pisarev, A. M. Balbashov, and T. Rasing, Nature (London) 435, 655 (2005).

[9] P. Nemec, E. Rozkotova, N. Tesarova, F. Trojanek, E. De Ranieri, K. Olejnik, J. Zemen, V. Novak, M. Cukr, P. Maly et al., Nat. Phys. 8, 411 (2012).
[10] F. Freimuth, S. Blügel, and Y. Mokrousov, Phys. Rev. B 94, 144432 (2016).

[11] F. Freimuth, S. Blügel, and Y. Mokrousov, Phys. Rev. B 92, 064415 (2015).

[12] K. M. D. Hals and A. Brataas, Phys. Rev. B 91, 214401 (2015).

[13] C. Ciccarelli, K. M. D. Hals, A. Irvine, V. Novak, Y. Tserkovnyak, H. Kurebayashi, A. Brataas, and A. Ferguson, Nat. Nanotechnol. 10, 50 (2014).

[14] H. Regensburger, R. Vollmer, and J. Kirschner, Phys. Rev. B 61, 14716 (2000).

[15] B. Koopmans, M. van Kampen, J. T. Kohlhepp, and W. J. M. de Jonge, Phys. Rev. Lett. 85, 844 (2000).

[16] T. J. Huisman, R. V. Mikhaylovskiy, A. Tsukamoto, T. Rasing, and A. V. Kimel, Phys. Rev. B 92, 104419 (2015).

[17] E. Beaurepaire, J.-C. Merle, A. Daunois, and J.-Y. Bigot, Phys. Rev. Lett. 76, 4250 (1996).

[18] B. Y. Mueller, A. Baral, S. Vollmar, M. Cinchetti, M. Aeschlimann, H. C. Schneider, and B. Rethfeld, Phys. Rev. Lett. 111, 167204 (2013).

[19] G. P. Zhang, M. S. Si, Y. H. Bai, and T. F. George, J. Phys.: Condens. Matter 27, 206003 (2015).

[20] M. Teichmann, B. Frietsch, K. Döbrich, R. Carley, and M. Weinelt, Phys. Rev. B 91, 014425 (2015). 
[21] K. Krieger, J. K. Dewhurst, P. Elliott, S. Sharma, and E. K. U. Gross, J. Chem. Theory Comput. 11, 4870 (2015).

[22] E. Carpene, H. Hedayat, F. Boschini, and C. Dallera, Phys. Rev. B 91, 174414 (2015).

[23] W. Töws and G. M. Pastor, Phys. Rev. Lett. 115, 217204 (2015).

[24] J. Wieczorek, A. Eschenlohr, B. Weidtmann, M. Rösner, N. Bergeard, A. Tarasevitch, T. O. Wehling, and U. Bovensiepen, Phys. Rev. B 92, 174410 (2015).

[25] A. J. Schellekens, N. de Vries, J. Lucassen, and B. Koopmans, Phys. Rev. B 90, 104429 (2014).

[26] I. L. M. Locht, I. Di Marco, S. Garnerone, A. Delin, and M. Battiato, Phys. Rev. B 92, 064403 (2015).

[27] F. Freimuth, S. Blügel, and Y. Mokrousov, J. Phys.: Condens. Matter 28, 316001 (2016).

[28] G. Géranton, F. Freimuth, S. Blügel, and Y. Mokrousov, Phys. Rev. B 91, 014417 (2015).

[29] J. C. Rojas Sanchez, L. Vila, G. Desfonds, S. Gambarelli, J. P. Attane, J. M. De Teresa, C. Magen, and A. Fert, Nat. Commun. 4, 2944 (2013).

[30] M. Isasa, M. C. Martínez-Velarte, E. Villamor, C. Magén, L. Morellón, J. M. De Teresa, M. R. Ibarra, G. Vignale, E. V.
Chulkov, E. E. Krasovskii et al., Phys. Rev. B 93, 014420 (2016).

[31] K. Shen, G. Vignale, and R. Raimondi, Phys. Rev. Lett. 112, 096601 (2014).

[32] S. Zhong, J. E. Moore, and I. Souza, Phys. Rev. Lett. 116, 077201 (2016).

[33] P. Ferriani, K. von Bergmann, E. Y. Vedmedenko, S. Heinze, M. Bode, M. Heide, G. Bihlmayer, S. Blügel, and R. Wiesendanger, Phys. Rev. Lett. 101, 027201 (2008).

[34] F. Freimuth, S. Blügel, and Y. Mokrousov, Phys. Rev. B 90, 174423 (2014).

[35] A. A. Mostofi, J. R. Yates, Y.-S. Lee, I. Souza, D. Vanderbilt, and N. Marzari, Comput. Phys. Commun. 178, 685 (2008).

[36] F. Freimuth, Y. Mokrousov, D. Wortmann, S. Heinze, and S. Blügel, Phys. Rev. B 78, 035120 (2008).

[37] C. Ciccarelli, L. Anderson, V. Tshitoyan, A. J. Ferguson, F. Gerhard, C. Gould, L. W. Molenkamp, J. Gayles, J. Zelezny, L. Smejkal et al., Nat. Phys. 12, 855 (2016).

[38] According to Ref. [16] the relative change of magnetization in Co amounts to $\Delta M / M \approx 0.1$ after 0.5 ps. Hence we estimate $\gamma \approx 0.1 /(0.5 \mathrm{ps})=2 \times 10^{11} \mathrm{~Hz}$. 